\title{
Case Files of the Medical Toxicology Fellowship at the University of Massachusetts Medical Center in Worcester, Massachusetts: Stumbling Through a Blue Haze
}

\author{
Richard J. Church, MD, Edward W. Boyer, MD, PhD
}

Division of Medical Toxicology, University of Massachusetts Medical Center, Worcester, MA

\section{CASE PRESENTATION}

A 56-year-old man with no significant medical history presented to a local emergency department (ED) with a complaint of foggy vision. He reported his eyesight was as if he were "walking through a smoky room." The patient reported that his symptoms began 1 day prior to arrival, approximately 1-2 hours after leaving a job site where he was working as a contractor. While at the work site, he had been in an enclosed room while another worker was applying polyurethane spray-on insulation to the walls. The patient had been wearing a paper mask that covered his mouth and nose, but no other protective gear.

\section{What is standard Personal Protective Equipment (PPE) and why do people wear it?}

Personal protective equipment (PPE) refers to items worn for protection from injury by chemical, biological, and electrical hazards and extremes of heat. The Environmental Protection Agency defines 4 levels of PPE, A through D, each providing differing degrees of protection depending on the hazards an individual may encounter (Table 1) [1]. When working with polyurethane spray-on insulation, guidelines for safe handling recommend the use of level A protection to reduce the risk of respiratory tract, skin or eye exposure to an uncontrolled amount of liquids or vapor [2].

\section{What are the components of polyurethane spray- on insulation products?}

The main constituents of all polyurethane spray-on insulation include polyols (compounds with multiple hydroxyl groups), isocyanates, and amines, which are often tertiary amines [3].

\begin{tabular}{|c|c|c|}
\hline Level & Equipment & Protection \\
\hline A & $\begin{array}{l}\text { SCBA, vapor-resistant, fully } \\
\text { encapsulated, airtight, } \\
\text { chemical-resistant suit } \\
\text { (permeation resistant) }\end{array}$ & $\begin{array}{l}\text { Gas, vapor, aerosol, liquid and } \\
\text { solid protection to respiratory } \\
\text { tract, mucous membranes, skin, } \\
\text { and eyes }\end{array}$ \\
\hline B & $\begin{array}{l}\text { Positive-pressure SAR, escape } \\
\text { SCBA, hooded, splash } \\
\text { protective, chemical-resistant } \\
\text { suit (splash suit) }\end{array}$ & $\begin{array}{l}\text { Gas, vapor, aerosol protective } \\
\text { to respiratory tract; liquid and } \\
\text { solid protection to skin and } \\
\text { eyes (no vapor protection) }\end{array}$ \\
\hline C & $\begin{array}{l}\text { APR, hooded, splash } \\
\text { protective, chemical-resistant } \\
\text { suit (splash suit) }\end{array}$ & $\begin{array}{l}\text { Select vapor and aerosol } \\
\text { protection to respiratory tract; } \\
\text { liquid and solid protection to } \\
\text { skin and eyes. }\end{array}$ \\
\hline D & Regular work clothing & None \\
\hline \multicolumn{3}{|c|}{$\begin{array}{l}\text { SCBAs (self-contained breathing apparatus): open-circuit, positive pressure devices } \\
\text { composed of a face mask connected to a compressed air source worn by the user } \\
\text { SAR (supplied-air respirator): positive-pressure device with air that is supplied through } \\
\text { a line from an outside source } \\
\text { APR (air-purifying respirator): mask that allows breathing of ambient air after } \\
\text { inhalation through a purifying canister or filter. There are } 3 \text { types: chemical cartridge, } \\
\text { disposable (N95), and powered-air (PAPR). }\end{array}$} \\
\hline
\end{tabular}

An example of a simple polyol is ethylene glycol. Each hydroxyl group in a polyol can participate in a chemical reaction, such as nucleophilic attack on a moiety such as an isocyanate. Hydroxyls combine with the $-\mathrm{N}=\mathrm{C}=\mathrm{O}$ functional group of isocyanates to produce urethanes. Successive reactions between diisocyanate and polyols produce, in a transformation known as a gel reaction, high-viscosity long-chain polyurethanes [4].

Keywords: glaucopsia, tertiary amines, chemical eye injury, blue haze

Notes: There was no outside funding of any kind used for this study.

Corresponding Author: Richard J. Church, MD, 55 Lake Avenue North, Worcester MA 02135. Email: churchr@ummhc.org 


\begin{tabular}{|c|c|c|}
\hline Morpholine & Dimethylethylamine & Tetramethylbutanediamine \\
\hline $\mathrm{N}$-methylmorpholine & Triethylamine & Ethylenediamine \\
\hline$N$-ethylmorpholine & Di-isopropylamine & Triethylenediamine \\
\hline Dimethylamine & 3-Dimethylaminopropylamine & tert-Octylamine \\
\hline Diethylamine & Tetramethylethylenediamine & Bis[2-(dimethylamino)ethyl]ether \\
\hline
\end{tabular}

Amines are a class of organic compounds derived from ammonia by replacing 1 or more of the hydrogen atoms with alkyl groups or nonacidic radicals containing hydrogen and carbon atoms. An amine is primary, secondary, or tertiary depending on whether 1,2 , or 3 of the hydrogen atoms of ammonia are replaced. Amines are used to catalyze the reaction between polyols and isocyanates. Catalysts are used to control both the gel reaction and the gas-forming reaction responsible for foam formation [2]. Tertiary aliphatic amines are widely employed as catalysts in polyurethane foam manufacturing operations [5]. Of the various amines that are used in the production of polyurethanes, some produce visual disturbances (Table 2).

\section{CASE CONTINUATION}

On evaluation, the patient appeared to be in no acute distress. His vital signs were: heart rate $62 / \mathrm{min}$; blood pressure 180/97 $\mathrm{mmHg}$; respiratory rate $16 / \mathrm{min}$; temperature $36.4^{\circ} \mathrm{C}$; and pulse oximetry $96 \%$ on room air. External examination of the patient's eyes showed very mild evidence of conjunctival irritation, without frank injection or excessive tearing. Visual acuity was 20/20 in the left eye and 20/15 in the right eye. Pupils were equal, round, and reactive to light and accommodation, each $4 \mathrm{~mm}$ in diameter with intact extraocular muscles. Funduscopic examination revealed no abnormalities, and slit lamp examination with fluorescein staining was unremarkable. Of note, during the examination the patient revealed that he was also noticing blue haloes around bright objects. The oropharyngeal mucous membranes were moist and not erythematous, and skin examination showed no evidence of irritation or rash. Cardiopulmonary examination revealed normal heart sounds without murmurs and clear lungs to auscultation bilaterally. The remainder of his physical examination was unremarkable.

\section{What are the basic mechanisms of color vision and visual acuity?}

Color vision depends on the ability to distinguish objects based on the wavelengths of the light they reflect or emit. The nervous system detects color by comparing the responses to light from the different types of retinal cone photoreceptors, each sensitive to different portions of the visible spectrum. For humans, the visible electromagnetic spectrum ranges from approximately 380 to $750 \mathrm{~nm}$. In the human eye, the cones are maximally receptive to short, medium, and long wavelengths of light and are called $\mathrm{S}-, \mathrm{M}-$, and L-cones, respectively. S-cones are sensitive to the portion of the visible spectrum wavelength from approximately 380 to $550 \mathrm{~nm}$, M-cones from 380 to $700 \mathrm{~nm}$, and L-cones from 425 to $750 \mathrm{~nm}$. The brain integrates the information from each type of receptor, giving rise to perception of different wavelengths of light [6]. Approximately 4.5 million cone photoreceptors in each retina allow for the high visual resolution experienced by humans.

The initial events in the perception of light-the point at which electromagnetic radiation is converted to chemical impulses in the central nervous system-revolve around rhodopsin, a G-protein coupled receptor family of retinal pigments that are extremely sensitive to light. Rhodopsin consists of the protein moiety opsin and a cofactor, retinal, which is synthesized in the retina from vitamin A and is reversibly bound via a Schiff base with an asparagine residue. Absorption of incident light by retinal induces an isomerization of 11-cis-retinal into all-trans-retinal, which produces a conformational change in opsin that converts rhodopsin into an activated form known as metarhodopsin II. Binding of the metarhodopsin II-associated G protein transducin triggers a second messenger cascade. This cascade involves the activation of phosphodiesterase (PDE)-6 that is necessary for the rapid catalysis of cGMP, leading to closure of the cGMP-gated cation channel of the photoreceptor, membrane hyperpolarization and transmission of the photoactivation signal [7]. Thus, the detection of light results from an interruption of the baseline frequency of action potentials generated by individual photoreceptors, contrary to the positive correlation between environmental input and neurologic signals, as would be intuited.

Visual acuity (VA) is the clarity of vision, which is dependent on the sharpness of focus within the eye, the concentration of photoreceptors, and the interpretative ability of the brain. To resolve detail, the eye's optical system has to project a focused image on the fovea, a region inside the macula having the highest density of cone photoreceptors, thus having the highest resolution and best color vision. Light travels from the object to the fovea through an imaginary path called the visual axis. The quality and color of the image perceived by the brain are affected by 


\begin{tabular}{|c|c|c|c|c|c|}
\hline Cornea & Iris & Lens & Retina & Optic Nerve & Optic Cortex \\
\hline Amiodarone & Bisphosphonates & Amiodarone & Hydroxychloroquine & Amiodarone & Carbon monoxide \\
\hline $\begin{array}{l}\text { Caustics } \\
\text { (acid, alkali) }\end{array}$ & Rifabutin & Corticosteroids & Methanol & Disulfiram & Cyanide \\
\hline Deferoxamine & Sulfonamides & Busulfan & Quinine & Chloramphenicol & Vasoconstrictors \\
\hline Irritants & Pamidronate & Chlorpromazine & Carbon disulfide & Ethambutol & Vasodilators \\
\hline \multirow{6}{*}{$\begin{array}{l}\text { Metals } \\
\text { (copper, gold, } \\
\text { mercury, silver) }\end{array}$} & & Iron & Deferoxamine & Isoniazid & Cisplatin \\
\hline & & & Digitalis & Linezolid & Cyclosporin \\
\hline & & & Ethambutol & Carbon tetrachloride & Tacrolimus \\
\hline & & & Thallium & Tetrachloroethylene & Interleukin \\
\hline & & & Vincristine & Toluene & $\begin{array}{l}\text { Methylmercury } \\
\text { compounds }\end{array}$ \\
\hline & & & & Lead & \\
\hline
\end{tabular}

tissues and structures in the eye in the visual axis. These structures include the cornea, anterior chamber, pupil, lens, vitreous humor, and finally the retina. The visual cortex is the part of the occipital cortex of the brain responsible for processing visual stimuli. The eyes are connected to the visual cortex by the optic nerves and optic tracts.

Any pathological process in the visual system will often cause decreases in visual acuity. Common causes of decreased visual acuity are disease processes that affect the visual axis (e.g., cataracts and corneal scarring), diseases that affect the retina (e.g., macular degeneration and diabetes), diseases affecting the optic pathway to the brain (e.g., tumors and multiple sclerosis), and diseases affecting the visual cortex (e.g., tumors and strokes) [8]. However, several xenobiotics also cause visual changes, either by affecting the absorption of ambient light or by damaging structures along the visual axis, the optic nerve, and optic cortex themselves (Table 3).

\section{What xenobiotics cause changes in color vision?}

A few xenobiotics produce derangements in color vision. Styrene affects color vision in a dose-dependent manner leading to dyschromatopsia, or selective color blindness, in the blue-yellow axis. Affected individuals thus have difficulty distinguishing between the colors yellow and blue. Styrene is a neurotoxic substance that binds to lipid-rich tissues such as brain, myelin, and adipose tissue, affecting neuron membrane receptors and interfering with the propagation of nervous impulses [9]. Although the neurochemical and structural bases of styrene neurotoxicity have not yet been elucidated (e.g., why does a chemical property such as lipid solubility affect only blue-yellow color perception?), this affect appears to be reversible when exposure decreases [10]. Ethambutol toxicity can lead to a loss of red-green discrimination, decreased visual acuity, as well as loss of peripheral vision.
These changes are due to the development of an optic neuritis that may be related to derangements in mitochondrial metabolism of copper [11]. Sildenafil citrate, often described as a "selective" inhibitor of PDE-5, also inhibits PDE-6, which is found in retinal photoreceptors. Inhibition of the PDE- 6 holoenzyme leads to persistently elevated levels of cGMP and depolarization of the cell membrane. This, in turn, causes photoreceptor cell degeneration in the retina and the characteristic blue vision, also known as cyanopsia, experienced by some individuals. Sildenafil also exhibits nonvisual ocular side effects that are uncommon, dosedependent, and reversible, including conjunctival hyperemia caused by retinal arteriolar dilatation via increased nitric oxide production $[12,13]$.

\section{How do amines cause changes in color vision?}

Tertiary amines have been used in foundries since the middle to late 1940s, and the hazards of exposures to these amines have been recognized in the scientific literature since their introduction. Mastromatteo identified several workers in Ontario exposed to heterocyclic amine catalysts who suffered from a condition described as "halo," "blue haze," or "foggy vision" [14]. Dernehl described blue haze as occurring in the vision of amine and foam plastic manufacturing personnel, and noted a diffuse corneal cloudiness that obscured the normal markings of the iris, as well as corneal edema with tiny vesicular collections of fluid on slit lamp examination [15]. Jones and Kipling later investigated several cases occurring in the manufacture of amines, the production of foam plastics, and from the use of amines in the cold curing process in foundries. Blue vision, grey vision, and haloes were the most frequently noted visual changes. Although no more precise data was made available, it was found that the lower molecular weight amines tended to produce a preponderance of blue vision and the higher molecular weight amines produced grey vision. 
The authors then coined the term "glaucopsia" from the Greek work glaucos, meaning blue-grey (see Table 2) [16].

Animal studies have been performed to determine which substance in polyurethane foams is chiefly responsible for the ocular pathology observed. In experiments in which vapors of tertiary amines and toluene diisocyanate (TDI) were directed at the corneas of anesthetized cats and monkeys, only tertiary amines led to denuded corneal epithelia, edema, areas of stroma and vesicles below the epithelium. In these studies, TDI was not found to be responsible for the corneal pathology; furthermore, the nonvolatile polyols were not considered to be possible causative agents [3].

In human experiments, healthy volunteers' eyes have been exposed to various airborne concentrations of different tertiary amines, causing pronounced visual disturbances including heavy hazing of the visual fields, lights surrounded by pronounced bluish halos, and slight eye discomfort. Ocular examination of study subjects revealed decreased visual acuity and contrast sensitivity, pronounced epithelial corneal edema, irregularities in the corneal epithelium, and subepithelial microcysts with increases in corneal thickness due solely to the epithelial edema, with no discernable damage to the endothelial layer [17]. Slight conjunctival injection of both eyes was also observed, strictly confined to the palpebral fissures [18]. The fact that the corneal edema and conjunctival irritation occurs only in the directly exposed part of the eye has led to the hypothesis that the effects of the amines are purely local with no systemic mechanism [5]. In addition, corneal opacity might result from diffusion of tertiary amines into the corneal epithelium; uncharged tertiary amines, with their 3 aliphatic groups, dissolve into the highly lipophilic corneal epithelium [19]. Furthermore, corneal edema and subepithelial microcysts are hypothesized to cause forward light scattering with subsequent blurring of vision. Mydriasis and paralysis of the ciliary muscles, leading to cycloplegia, have also been reported by individual investigators as contributors to visual changes $[17,20,21]$.

Higher ambient air concentrations of total amines are associated with increased risk of blurry vision, halo vision and blue-grey vision. The risk of corneal opacity also rises with increasing concentration and duration of exposure to total amines [19].

Ballantyne described a continuum of progression of symptoms arising from amine exposure. Initial symptoms include haziness of vision, followed by a more marked difficulty with visual discrimination accompanied by a partial loss of color discrimination, resulting in objects taking on a grey or blue color. Moderate exposures are characterized by the development of a halo effect appearing around bright objects. In more severe cases photophobia occurs. One of the earliest indications of glaucopsia may be an impairment of ability to visualize written text correctly [22]. Other symptoms associated with tertiary amines are typically related to inhalational exposure and include irritation of the trachea and bronchi, headaches, nausea, and lightheadedness $[23,24]$.

\section{When do symptoms begin and how long do they last?}

The concentration of amine in the atmosphere will influence the time to onset and progression of symptoms; at greater airborne amine concentrations, the individual may experience irritation to the eye characterized by stinging and reddening. Exposures to concentrations higher than those resulting in glaucopsia are associated with peripheral sensory irritant effects and inflammatory changes. The level of these concentrations can vary widely depending on the amine involved. This observation has led to the conclusion that glaucopsia represents the earliest and most sensitive effect of amines on the eye [22]. Effects begin 1-2 hours after initial exposure, with symptoms lasting anywhere from a few hours to a few days after cessation of exposure to the causative vapor.

\section{How does one adequately evaluate the visual changes caused by amines?}

Evaluation of the eye should begin with visual acuity, often referred to as the vital sign of the eye. However, in mild cases of corneal edema secondary to chemical exposures, such as to tertiary amines, standard visual acuity testing is often normal and any impairment can be documented only by measurement of contrast sensitivity [22]. Contrast sensitivity refers to the crispness of vision, that which enables individuals to see objects that do not stand out from their backgrounds. It is often referred to as "functional vision" beyond how well one sees details on a standard high contrast (black on white) eye chart. Many contrast sensitivity tests use bar or dot patterns displayed against backgrounds of varying contrast. These objects are shown at varying intensities, making them easier or more difficult to detect. The measurement of visual acuity at low contrast may show deterioration in vision when high contrast tests fail to measure subjective visual symptoms. With low contrast sensitivity, there are problems with night driving and individuals may require extra light to read. Low contrast sensitivity also can be a symptom of certain eye conditions or diseases such as cataracts, glaucoma, or diabetic retinopathy. Also, reduced contrast sensitivity sometimes can occur as a usually temporary side effect of laser vision surgery procedures [25].

Slit lamp examination with and without fluorescein staining is another important component in the evaluation of the eye. This procedure allows for the detection of corneal opacity and corneal defects such as abrasions, laceration, and ulcers. Much of the ocular pathology observed in tertiary amine exposure can be seen on slit lamp examination, including conjunctival injection (often confined to the palpebral fissures), subepithelial microcysts, corneal surface irregularities, and corneal edema [5]. Cloudiness of the cornea due to edema can result in blurring of iris margins, another finding notable on slit lamp examination [17].

Corneal pachymetry is the measurement of the thickness of the cornea. This test can be performed using an optic Haag-Streit pachymeter or an ultrasonic pachymeter. This test is important in the early detection of glaucoma and in ensuring sufficiency of 
corneal thickness prior to laser-assisted in situ keratomileusis (LASIK) surgery. However, it is sensitive enough to detect the small increase in corneal thickness caused by the edema confined to the corneal epithelial cells due to tertiary amine exposure $[17,26]$.

\section{CASE CONTINUATION}

The patient reported that he had contacted the insulation product manufacturer and was told he was suffering from "Blue Haze Syndrome" and that his symptoms would be better by the next morning. However, when he was still having difficulty with his vision, he presented to the ED.

The consulting toxicologist also contacted the insulation company and was directed to an ophthalmologist who had seen several employees of the insulation product company for the same constellation of symptoms. A detailed explanation of the pathology and pathophysiology of "Blue Haze" was described, and instructions on treatment and prognosis were made.

The patient was instructed to follow up with an ophthalmologist. However, upon follow-up the next day by telephone with the toxicologist, he had reported his symptoms had completely resolved and ultimately he did not get examined by an ophthalmologist.

\section{What is the standard treatment for Blue Haze?}

Initial treatment for any eye exposure is removal of the offending agent. Prompt irrigation at an eyewash station and removal of contact lenses help to prevent continued exposure. The phenomenon of glaucopsia has been described as a transient disturbance of vision that leaves no permanent ocular sequelae [22]. No reports of individuals suffering irreversible eye damage even after long-term exposure have been published. Once removed from the exposure, the thickness of the cornea has been found to gradually return to normal, leading to restored vision [21]. Due to the spontaneous resolution of objective and subjective findings, no role for medical treatment has been discussed or implemented. Often, individuals are seen by medical professionals long after their symptoms have resolved and are only able to give a retrospective history.

More important, perhaps, is the heightened level of risk that has been connected to these disturbances in vision. Glaucopsia can be considered a hazard as it may impair work efficiency and predispose individuals to physical accidents as well as hinder performance of coordinated tasks. The visual changes pose a real threat when driving vehicles, and several workers in many case studies have complained of difficulty in discriminating traffic signals while driving, especially at night $[19,24]$.

\section{CONCLUSION}

Glaucopsia, or "Blue Haze," is a transient disturbance of vision resulting from exposure to the vapor of certain industrial chemicals, notably amines that can be found in products such as spray-on polyurethane foams. Visual effects such as halos around bright objects, blue-grey vision, and fogging of vision typically appear within a few hours of exposure and resolve without permanent sequelae a few hours after removal of the exposure agent, although symptoms have been known to last up to 2-3 days. Effects are due to local corneal contamination leading to epithelial edema with associated microcysts that cause forward light scattering. The changes in vision can predispose individuals to accidents at the workplace as well as while driving. Measures to reduce or completely prevent ocular exposures to tertiary amines include wearing the proper personal protective equipment, avoiding wearing contact lenses that can act as reservoirs for amines, limiting exposure times, and keeping workplaces well ventilated to reduce vapor concentrations $[17,22]$.

The authors have no potential financial conflicts of interest to report.

\section{REFERENCES}

1. Walter FG. Hazmat incident response. In Flomenbaum NE, Goldfrank LR, Hoffman RS, et al., eds. Goldfrank's Toxicologic Emergencies, New York: McGraw-Hill, 2006:1764-1774.

2. American Plastics Council. Polyurethane amine catalysts: guidelines for safe handling and disposal. Alliance for the Polyurethane Industry Technical Bulletin 2000:1-16.

3. Potts AM, Rouse EF, Eiferman RA, et al. An unusual type of keratopathy observed in polyurethane workers and its reproduction in experimental animals. Am J Ind Med 1986;9:

203-213.

4. Seo D, Youn JR. Numerical analysis on reaction injection molding of polyurethane foam by using a finite volume method. Polymer 2005;46(17):6482-6493.

5. Stahlbom B, Lundh T, Floren I, et al. Visual disturbances in man as a result of experimental and occupational exposure to dimethylethylamine. Br J Ind Med 1991;48: 6-29.

6. Rowe MH. Trichromatic color vision in primates. News Physiol Sci 2002; 7(3):93-98.

7. Cote RH. Photoreceptor phosphodiesterase (PDE6): a Gprotein-activated $\mathrm{PDE}$ regulating visual excitation in rod and cone photoreceptor cells. In: Beavo JA, Francis SH, Houslay MD, eds. Cyclic Nucleotide Phosphodiesterases in Health and Disease, Boca Raton, FL: CRC Press; 2006:165-193.

8. Cline D, Hofstetter HW, Griffin JR. Dictionary of Visual Science. 4th ed. Boston: Butterworth-Heinemann; 1997:147-160.

9. Gong YY, Kishi R, Katakura Y, et al. Relation between colour vision loss and occupational styrene exposure level. Occup Environ Med 2002;59:824-829.

10. Iregren A, Andersson M, Nylen P. Color vision and occupational chemical exposures. I. An overview of tests and effects. Neurotoxicol 2002;23:719-733.

11. Kozak S, Inderlied C, Hsu H, et al. The role of copper on ethambutol's antimicrobial action and implications for 
ethambutol-induced optic neuropathy. Diag Microbiol Infect Dis 1998; 30:83-87.

12. Jägle H, Jägle C, Sérey L. Visual short-term effects of Viagra: double-blind study in healthy young subjects. Amer J Ophthalmol 2004;137(5):842-849.

13. Yuan Z, Hein TW, Rosa RH, et al. Sildenafil (Viagra) evokes retinal arteriolar dilation: dual pathways via NOS activation and phosphodiesterase inhibition. Invest Ophthalmol Vis Sci 2008;49: 720-725.

14. Mastromatteo E. Recent occupational health experiences in Ontario. J Occup Med 1965;7:502-511.

15. Dernehl CU. Health hazards associated with polyurethane foam. J Occup Med 1966;8:59-62.

16. Jones WT, Kipling MD. Glaucopsia-blue-grey vision. $\mathrm{Br} J$ Ind Med 1973;29:460-461.

17. Jarvinen P, Engstrom K, Riihimaki V, et al. Effects of experimenta exposure to triethylamine on vision and the eye. Occup Environ Med 1999;56:1-5.

18. Akesson B, Floren I, Skerfving S. Visual disturbances after experimental human exposure to triethylamine. $\mathrm{Br} J$ Ind Med 1985;42:848-850.
19. Page EH, Cook CK, Hater MA, et al. Visual and ocular changes associated with exposure to two tertiary amines. Occup Environ Med 2003;60:69-75.

20. Albrecht WN, Stephenson RI. Health hazards of tertiary amine catalysts. Scand J Work Environ Health 1988;14(4):209-219.

21. Reilly MJ, Rosenman KD, Abrams JH, et al. Ocular effects of exposure to triethylamine in the sand core cold box of a foundry. Occup Environ Med 1995;52:337-343.

22. Ballantyne B. Glaucopsia: an occupational ophthalmic hazard. Toxicol Rev 2004;23(2):33-90.

23. Brieger H, Hodes WA. Toxic effects of exposure to vapors of aliphatic amines. Arch Ind Hyg 1951;3:287-291.

24. Yoshida T, Ono Y, Muto S, et al. Visual disturbances among workers exposed to triethylamine in a foundry in Japan. J Occup Health 2001;43:199-200.

25. Jarvinen P, Hyvarinen L. Contrast sensitivity measurement in evaluations of visual symptoms caused by exposure to triethylamine. Occup Env Med 1997;54:483-486.

26. Akesson B, Bengtsson M, Floren I. Visual disturbances after industrial triethylamine exposure. Int Arch Occup Env Health 1986;57(4):297-302. 